\title{
Assessing Polymorphism Information Content (PIC) Using SSR Molecular Markers on Local Species of Citrullus Colocynthis. Case Study: Iran, Sistan-Balouchestan Province
}

\author{
Sattar Enayat Avval ${ }^{1}$ \\ ${ }^{1}$ Department of Biology, Genetics, Faculty of Sciences, University of Zabol, Zabol, Iran \\ Correspondence: Sattar Enayat Avval, Department of Biology, Genetics, Faculty of Sciences, University of Zabol, \\ Zabol, Iran.
}

Received: February 4, 2017

Accepted: February 16, 2017

Online Published: March 8, 2017

doi:10.5539/jmbr.v7n1p42

URL: https://doi.org/10.5539/jmbr.v7n1p42

\begin{abstract}
Studying polymorphism information content in plants, particularly local plants which are rich in genetic variety, can play an efficient role in building genetic bank and species' breeding. Iranian colocynth is highly important in terms of medicinal and therapeutic traits and hence, it needs assessing the polymorphism information content. In present research, simple sequence repeat (SSR) markers are used. 32 samples are randomly collected from local accumulations in 8 regions of Sistan-Balouchestan province. DNA extraction from each sample was done using Cetyltrimethylammonium Bromide. 10 primers were designed and used in this study. Polymerase chain reaction was done using extracted DNA and ten primers. To analyze genetic data, NTSYS pc ver.2/2, Genalex 6.5 and XLSATAT software were used. Regarding data analysis of respective values and taking this fact into account that 7 out of 10 used primers showed polymorphism information content in respective samples, their classification was not possible and hence, categorization of similar shapes was not performed due to highly genetic diversity of this species and, also, each value had a different shape.
\end{abstract}

Keywords: polymorphism, colocynth, molecular marker, SSR

\section{Introduction and Objective}

Due to high genetic diversity of flora, specially, in essence-containing and medicinal plants, Iran provides a nice ground for exploiting advanced facilities for breeding plans. Plants diversity is enormous due to the variable climates in Iran which causes changes in sub-species. This diversity is immensely important for present and future studies of Iran and the world (Moghadam, 1998). The primary source of colocynth is archipelago located in southern areas of Bhutan (Gichimu et al. 2009; Huh et al., 2008). It is scientifically called Citrullus Colocynthis and belongs to Cucurbitaceae family. It's a perennial Herbaceous plant with climbing or bended stalk coated with fiber scattered in different desert areas or wastelands and among sand hills. Its leaves are divided into seedy lobes coated with fibers in a relatively white appearance on the bottom surface of lamina. Ivies come out from both sides of its leaves which connect it to the staddle. Its flowers are in single position divided into male and female genera on a joint base. This plant is categorized in medicinal plants group (Imami, Ahi, 1387). The shell of this fruit is used as preservative, jelly, conserve and also pickles (Dene et al., 2004\&2007). The medicinal effects of this plant is to such extent that have been the subject of many studies in Iran and other parts of the world such as Afifia et al., 1973; Wasfi, 1994; Darwish et al., 1974; Barth et al., 2002 and TavakolAfshari et al., 2005.

In a study by Lee et al (1996), SSR markers were used to determine genetic diversity among PI achievements of CitrullusLanatusvarLanatus, CitrullusLanatusvarCitroides and wild species of CitrullusColocynthis and 4 groups were described in genetic level of $25 \%$. Molecular markers have a wide variety in terms of herbal molecular biological and biotechnological functions (Kumar et al., 1999, 2009). Jerret et al. (1997), in a study based on using RAPD molecular markers among 46 inherited cultivators of watermelon, concluded that there is a low genetic diversity and that cultivated watermelon is very limited in genetic base. Levi et al. (2001) evaluated RAPD genetic diversity in Pls and divided them into two groups of Citrullus LanatusvarCitroides and Citrullus Colocynthis.

Since there are SSR molecular markers in the genome of plants such as Colocynth and they have various sizes in terms of length, and also they have advantages of being Locus-specific and being codominant and capability of 
being assessed by PCR molecular and highly Polymorphic technique, so, we used such molecular markers to study polymorphism in local colocynth accumulations in the region.

The objective of this research is to reveal the possible relationship between morphology and respective genetic polymorphism area of local colocynth accumulations. Since Citrullus Colocynthis wildly grows in pasturelands across Sistan-Balouchestan province, its morphological evaluation was studied. Guo et al. (2012) assessed isolation and description of specific characteristics of 10 SSR markers in their study. Specific characteristics of 10 new polymorphic microsatellite Locuses were obtained for bitter melon. Polymorphism levels in these microsatellite Locuses suggest their useful capability for being used in genetic diversity evaluations, molecule fingerprint, identification, comparative genomic analysis and genetic planning in Momordica species (Guo et al., 2012). In another study, Levi et al. (2001) assessed genetic and kinship diversity among 90 CytrolusLanatus watermelons. Totally, 201 fragments were created of 30 compounds. Among these achievements, only 87 fragments were polymorphic. Therefore in this research, there was polymorphism of $43 \%$. Ghanavati (2014) evaluated morphologic molecular diversity of different Iranian colocynth accumulations. In this study, RAPD molecular marker was used for all 10 starters, polymorphism production strips and 137 produced bands showed polymorphism phenomenon of $99 / 16 \%$. In 2015 , Wang and his colleagues assessed genetic diversity of Thumb Masturm watermelon in China and used two molecular markers to assess sequence-related amplified polymorphism (SRAP) and simple sequence repeats (SSR). The results indicated high correlation of SRAP and SSR to the extent of 0.86 which points out the genetic closeness of internal and external amounts. Also, morphologic and polymorphism diversity were observed.

\section{Materials and Methods}

\subsection{Materials: Colocynth}

\subsection{Method of Operation}

\subsubsection{Sampling}

Samples were randomly collected from 8 regions of Sistan-Balouchestan province (Iran), 4 samples from each and in sum, 32 samples were collected. These 8 regions include Khash, Irandegan, Zahedan, Iranshahr, Kheirabad, Dustmohammad, Bampour and Daman. The samples were taken in terms of morphological characteristics and the place of collecting, and after being put in a plastic nylon, were kept and transferred to laboratory in an ice tank at a Sub-zero temperature. Afterwards, the samples were kept in freezer at the temperature of minus $80^{\circ} \mathrm{C}$ in order to extract DNA and to do molecular evaluations.
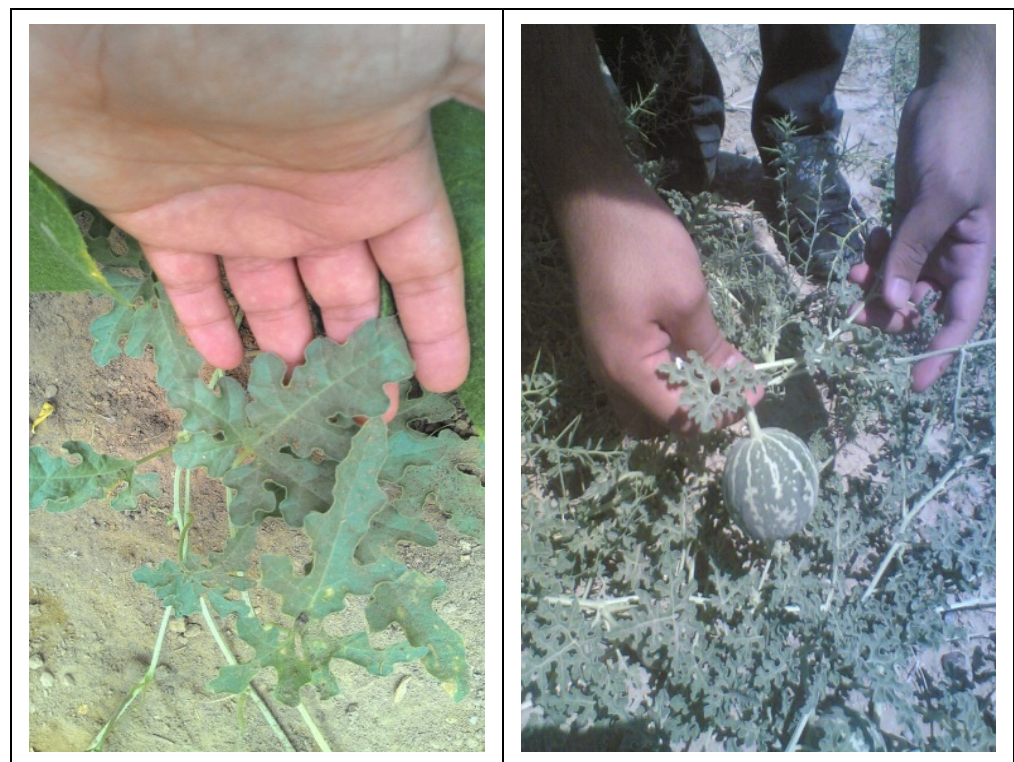

Picture 1. Morphological characteristics of colocynth (Citrullus Colocynthis) in natural habitats of SistanBalouchestan 


\subsubsection{Extracting Genomic DNA}

In order to extract genomic DNA from colocynth's leaf, genomic extraction kit of Fermentas (\#K0512) was used as follows:

1) Firstly, $100 \mathrm{mg}$ of fresh leaves were placed in liquid nitrogen and then were chopped using a pounder and became very small particles.

2) We poured plant powder into the $1.5 \mathrm{ml}$ vial and dissolved it in $200 \mu \mathrm{l}$ of TE buffer.

3) In the next step, we added $400 \mu$ of kit's lysis buffer to the sample and incubated it for $5 \min$ in $65^{\circ} \mathrm{C}$.

4) $600 \mathrm{Ml}$ of chloroform was immediately added and then we inverted the vial slowly for a few times for it to be mixed.

5) It was centrifuged at $9500 \mathrm{~g}$ for 2 minutes.

6) The top phase containing the DNA was transferred to a new vial and $800 \mu \mathrm{l}$ of sedimentary buffer included in the kit was add to it and they were mixed by slowly inverting the vial for a few times.

7) It was centrifuged at $9500 \mathrm{~g}$ for 2 minutes.

8) The top phase was completely removed. Sediment of DNA was dissolved in $100 \mu \mathrm{l}$ of $\mathrm{NaCl}$ salt (slowly).

9) $300 \mu \mathrm{l}$ of cold ethanol were added to it and then it was put at a temperature of $-20^{\circ} \mathrm{C}$ for 20 minutes in order for DNA to be deposited.

10) It was centrifuged at $9500 \mathrm{~g}$ for 4 minutes. Ethanol was removed and the sediment was washed in $300 \mu \mathrm{l}$ of $70 \%$ cold ethanol.

It was centrifuged at $9500 \mathrm{~g}$ for 4 minutes. Ethanol was removed and the sediment was slowly dissolved in $100 \mu \mathrm{l}$ of sterile twice distilled water.

Then, to determine density and purity of extracted DNA of the samples, Nano-drop instrument was used. A part of final solution was taken for electrophoresis. In the next step, electrophoresis was done.

\subsubsection{Primer}

In order to do molecular evaluations of genetic sequences diversity using SSR technique, we used primers to proliferate known microsatellite sequences of colocynth based on the sources. 10 primers were designed and bought from the company of Yekta Tajhiz Azma and the names of each of the primers were obtained from the paper of J. M. GUERRA-SANZ (doi:10.1046/j.1471-8286 2002.00200.x). Therefore, ten pairs of primers used in this research are represented in Table 1.

Table 1. Ten pairs of primers used in SSR technique

\begin{tabular}{|c|c|c|}
\hline Position and succession of the primer & Primer's name & Pair \\
\hline $\begin{array}{l}\text { ADPGPS-F: ACCACAACACAACACAACAC } \\
\text { ADPGPS-R: AAGCTTTGCATTTACTTCCA }\end{array}$ & AF032471.1 & 1 \\
\hline $\begin{array}{l}\text { SAT2-F: AGATTGACAACACATGCAGA } \\
\text { SAT2-R: TTTTGCTCATCCTACGAGAT }\end{array}$ & D85624.1 & 2 \\
\hline $\begin{array}{l}\text { SAT3-F: TGAGAGGAAAGGAACCATAA } \\
\text { SAT3-R: GTCTCTTGCAAAGCTCAAACT }\end{array}$ & AB006530.1 & 3 \\
\hline $\begin{array}{l}\text { URF1-F: AGCAGCACCTTGTCTTGTAT } \\
\text { URF1-R: CACAGATCCCACTCAATCTT }\end{array}$ & X04130.1 & 4 \\
\hline $\begin{array}{l}\text { MITODIXI-F: ATCTGTCAAGATCACGAAGG } \\
\text { MITODIXI-R: TGGGAAATAGGAAACGTAGA }\end{array}$ & AF288042.1 & 5 \\
\hline $\begin{array}{l}\text { WGA3H-F: CGATAATCGACTTTCTCGAC } \\
\text { WGA3H-R: GCTTCTTAGCATCATCAACC } 2\end{array}$ & AF074710.1 & 6 \\
\hline $\begin{array}{l}\text { EST00675-F: CTTCCTTTCTCTTCATTCCC } \\
\text { EST00675-R: TGAGGGAAAACGAGTTTAGA }\end{array}$ & EST00675 & 7 \\
\hline $\begin{array}{l}\text { EST00667-F: ATTGACTCTGATTCTCCCG } \\
\text { EST00667-R: GGAGGAAGATCAAAAGAACC }\end{array}$ & EST00667 & 8 \\
\hline $\begin{array}{l}\text { EST00507-F: CTTGTTTTCTTTTTCGATGG } \\
\text { EST00507-R: ATTAGATGGATGGTGTCAGC }\end{array}$ & EST00507 & 9 \\
\hline $\begin{array}{l}\text { WSUS-F: ATGAGTGAGAAAGCTTGGAA } \\
\text { WSUS-R: AATACTCCAATTGATGACGG }\end{array}$ & AB018561 & 10 \\
\hline
\end{tabular}




\subsubsection{Polymerase Chain Reaction (PCR)}

We used polymerase chain reaction to assess molecular genetic variety in microsatellite sequences using SSR markers. Afterwards, the samples were set up and appropriate thermal cycles were determined based on primers (Table 2 and 3). Then, electrophoresis was performed with Polyacrylamide gel within the samples were run after PCR on $8 \%$ polyacrylamide gel for 2 hours and 15 minutes with the voltage of 80 to 100 (amps vary but are about 30 to 60 ) and were colored with ethidium bromide and were observed by Gel Doc and their images were taken (Table 4).

Table 2. The Primers

\begin{tabular}{|c|c|c|}
\hline Primer's name & $\mathrm{Tm}$ & $\mathrm{Ta}$ \\
\hline \multirow[t]{2}{*}{ WSUS } & $\mathrm{F}=48.6$ & 43.5 \\
\hline & $\mathrm{R}=48.7$ & \\
\hline \multirow[t]{2}{*}{ MITOD } & $\mathrm{F}=48.8$ & 43.5 \\
\hline & $\mathrm{R}=48.5$ & \\
\hline \multirow[t]{2}{*}{ SAT3 } & $\mathrm{F}=48.5$ & It was examined at the temperature 43 to 50 \\
\hline & $\mathrm{R}=50.9$ & and also 55 and no results were obtained. \\
\hline \multirow[t]{2}{*}{ EST00507 } & $\mathrm{F}=49.1$ & 44 \\
\hline & $\mathrm{R}=48.9$ & \\
\hline \multirow[t]{2}{*}{ ADPGPS } & $\mathrm{F}=47.9$ & 46.2 \\
\hline & $\mathrm{R}=49.3$ & \\
\hline \multirow[t]{2}{*}{ URF1 } & $\mathrm{F}=48.3$ & 48 \\
\hline & $\mathrm{R}=48.6$ & \\
\hline \multirow[t]{2}{*}{ EST00675 } & $\mathrm{F}=49.3$ & 44.5 \\
\hline & $\mathrm{R}=48.9$ & \\
\hline \multirow[t]{2}{*}{ WGA3H } & $\mathrm{F}=48.7$ & 46.8 \\
\hline & $\mathrm{R}=48.7$ & \\
\hline \multirow[t]{2}{*}{ EST00667 } & $\mathrm{F}=48.9$ & 43.5 \\
\hline & $\mathrm{R}=49.1$ & \\
\hline \multirow[t]{2}{*}{ SAT2 } & $\mathrm{F}=48.7$ & 45 \\
\hline & $\mathrm{R}=48$ & \\
\hline
\end{tabular}

Table 3. The values used for PCR test

\begin{tabular}{ll}
\hline primer & One lambda from each \\
\hline purified DNA sample & One lambda \\
Master Mix & 10 lambdas \\
water & 7 lambdas \\
\hline
\end{tabular}

Table 4. The volume of required materials to produce Gel

\begin{tabular}{llllll}
\hline Gel\% & $30 \%$ Acrylamide $(29: 1)$ & $\mathrm{H} 2 \mathrm{O}(\mathrm{ml})$ & $5 \mathrm{x}$ TBE $(\mathrm{ml})$ & $10 \%$ APS $(\mu \mathrm{l})$ & TEMED $(\mu \mathrm{l})$ \\
\hline $8 \%$ & $3.2 \mathrm{ml}$ & 6.4 & 2.4 & 200 & 10 \\
\hline
\end{tabular}

\subsection{Data Analysis}

We scanned electrophoresed gels using UVDOC instrument and their images were used to extract data. Bands were given marks in positions for each sample according to being binary or non-binary for each marker. Then, we extracted the data from the gel such that if there was a band marker, mark1, and if there was no position for an allele in that sample, mark 0 was given.

Marker's polymorphic information content (PIC) which shows that the polymorphism of the marker can be a relative amount ranging from 0 to 1 ; the bigger the number is, the greater the variety of alleles in the studied place will be. In this regard, there is a probability of identity (PI) which indicates the probability of two distinct numbers being separated by a single marker, which isPIC $=1-\left(\sum P I\right)^{2}$. Also, PCOA analysis was used to confirm the categorization. 


\section{Results}

\subsection{Polymorphism Obtained from SSR}

All samples were set up and thermal gradient PCR was applied on each of the primers. Each primer was evaluated at different temperatures. After PCR with primers, samples were run and colorized on Agarose gel (pictures 2 and $3)$.

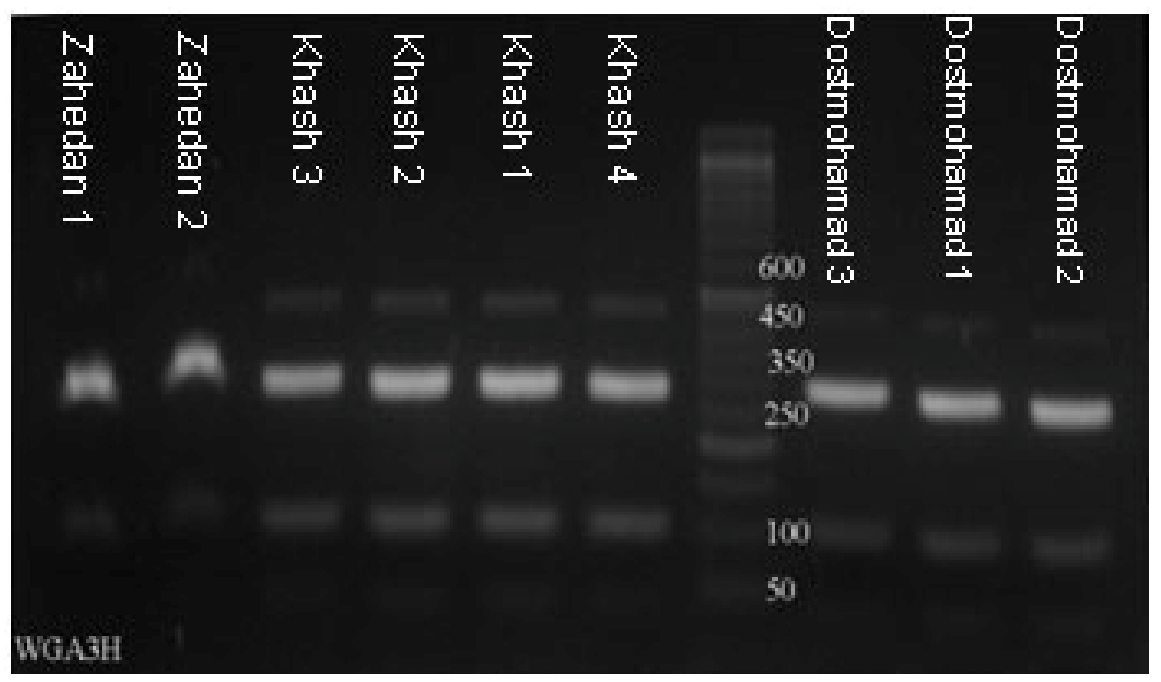

Picture 2. Agarose gel containing samples collected from Zahedan, Khash and Dustmohammad reproduced by WGA3H primer during PCR

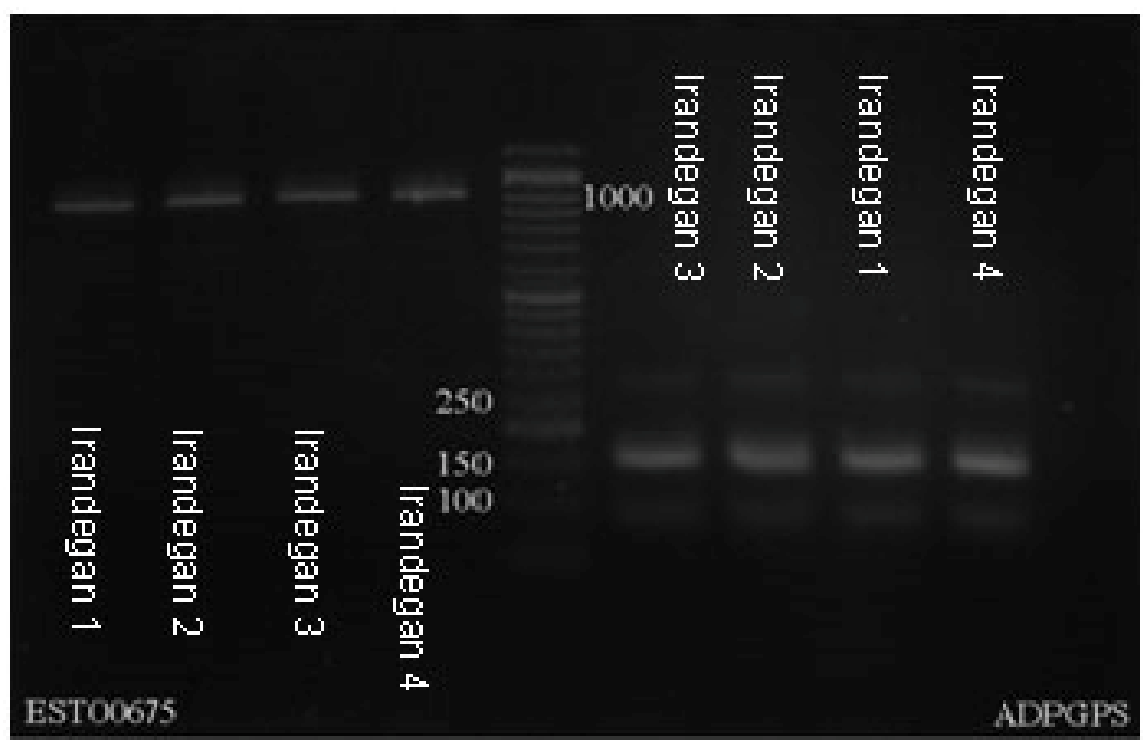

Picture3. Agarose gel containing samples collected from Irandegan reproduced by EST00675 and ADPGPS primers during PCR

\subsection{Polymorphic Information Content}

By using alleles' frequency for each starter, the polymorphic information content (PIC) was calculated. PIC value ranges from 0 to 1 ; the closer this amount to PIC is, the greater allele's reproduction frequency with that starter will be. 
The greatest value of PIC is of the ADPGPS primer $(0 / 85)$. In the second position, it was WGA3H primer. Each starter containing greater PIC would be a better choice for determining genetic variety. As a matter of fact, selectivity would be possible for future researches. Therefore, regarding polymorphic information contents of ADPGPS and WGA3H primers are recommended for further studies.

In Table 5, PIC of the primers used in this study is indicated. Figure 1 diagrams the percentage of unique bands which are the genetic location (Locus) in the primers used.

The obtained results from SSR marker are summarized in Table 6.

Table 5. PIC of the primers used in this study

\begin{tabular}{ll}
\hline Primer's name & PIC \\
\hline WSUS & 0 \\
MITOD & 0 \\
EST507 & 45.32 \\
ADPGPS & 0.85 \\
URF1 & 0.48 \\
EST00675 & 68.75 \\
WGA3H & 0.82 \\
EST00667 & 0.36 \\
SAT2 & 0.5 \\
\hline
\end{tabular}

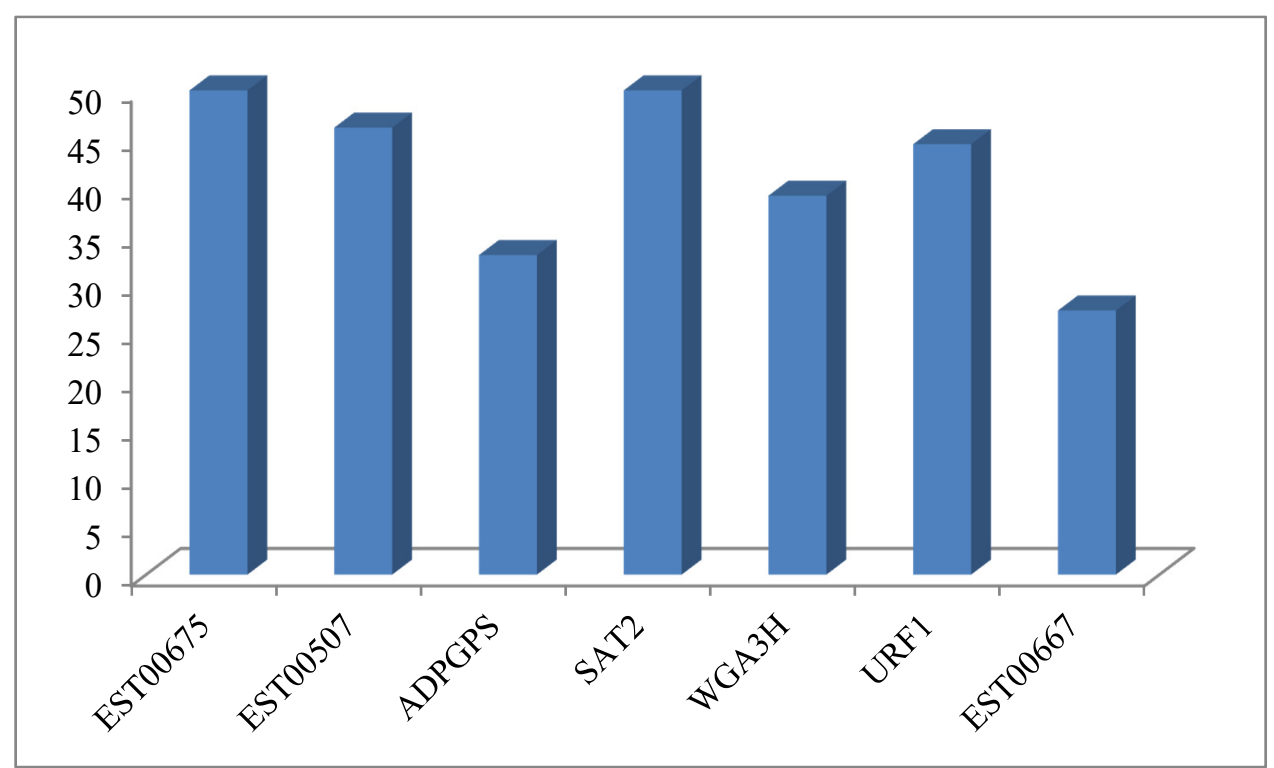

Figure 1. the diagram of percentage of unique bands which are the genetic locations (Locus) in the primers used

Table 6. summary of obtained results from SSR marker

\begin{tabular}{ll}
\hline The total number of bands & 102 \\
\hline The number of reproduced locations & 40 \\
The number of reproduced polymorphic locations & 100 \\
The average number of each primer's bands & 14 \\
The primer with the greatest polymorphic value & ADPGPS \\
The primer with the least polymorphic value & EST00667 \\
The primer with the highest number of bands & ADPGPS \\
& WGA3H \\
The primer with the least number of bands & EST00667 \\
& EST00675 \\
\hline
\end{tabular}




\subsection{Principal Coordinates Analysis}

Principal coordinates analysis is a method of reducing data in order to determine the relationship between several independent characteristics among genetic samples. To do principal coordinates analysis, GenAlex 6.5 and XLSTAT software were used. The results emphasized on the existing of variety among samples and the necessity of using more primers to categorize samples in terms of similarity and relationships of genetic variety.

\section{Discussion}

Since this study did not contain 3 polymorphic primers and was performed using 7 primers despite assessing resources and selectivity of the primers, lack of success in categorizing samples can be due to the high variety in the study's accumulations and the necessity to include more primers in classification procedure. Although in many researches of this kind, choosing 10 primers using sources evaluation method is common, in a research with the subject of isolation and determination of 10 SSR markers of Momordica Charantia whose results were published in 2012, SSR molecular markers were used in the studies about genetic variety and population structure of bitter melon (Guo et al., 2012).

So, we can assume that the great variety in samples is the major cause of impossibility of samples' categorization in terms of genetics. But morphological evaluations during plant sampling, also, showed morphological variety among samples collected from different regions.

In a study by Wang et al. (2015), they studied genetic diversity and population structure of Momordica Charantia among 4 species namely Cucumissativus, LagenariaSiceraria L., Luffa Cylindrical L. and Cucurbita Pepo L.in order to enhance and determine microsatellite polymorphic markers in Momordica Charentia plant. In their study, 16 microsatellite polymorphic Lucuses were assessed in 36 plants. 7 out of 16 pairs of primers were reproduced successfully using PCR in 4 respective species.

The issue of Citrullus colocynthis having a special genetic variety compared to other Citrullus genera is confirmed by comparative analysis related to chloroplast genome diversity of pasture and farming species of Citrullus genus by Dane et al. (2003). Hence, this is an emphasis on the importance of performed researches about finding and selectivity of the genetic diversity studies of this genus; but the accomplished researches related to colocynth are rare compared to the amount of cultivated watermelon. Therefore, it seems that the necessity of doing such research about this plant should be considered (Levi et al., 2001; IpekUluturk et al., 2011). Although one theory emphasizes on the issue of cultivated watermelon being derived from colocynth (Dane et al., 2007), the genome of colocynth has still a unique genetic diversity which needs more considerations because colocynth is highly useful and important in terms of therapeutic applications (Goldfain et al., 1989).

The results of this study which were obtained from assessing SSR marker in 32 collected samples from 8 randomly selected regions in Sistan-Balouchestan province emphasize on a great genetic diversity in local accumulations. Despite this issue that 7 out of 10 primers used in this research showed polymorphism in respective samples, it was not possible to classify them. Therefore, the primers were well chosen for local colocynth accumulation. Classification depends on more considerations using more primers and choosing them well in order to categorize local accumulations through genetic polymorphism. So, this study's results confirm great genetic diversity in Citrullus Colocynthis in the samples which seems to be limited to Sistan-Balouchestan, the whole country (Iran) and Iranian plateau. Therefore, several recommendations based on obtained results are presented as follows.

\section{Recommendations}

Regarding the variety observed in each marker, it seems that despite using appropriate primers in terms of resources, and because colocynth local accumulations contain unique variety, more primers are required to examine genetic variety of collected samples in order to eventually reach a well-chosen set of primers for genetically categorizing Citrullus Colocynthis. According to Levi et al. (2001b) it is recommended to use SRAP marker which is one of the efficient strategies in determining polymorphism in crops such as watermelon containing low levels of polymorphism. So, considering existent variety, it is recommended to perform this research in the whole-country scale to provide a gene bank containing this species' germ plasm. Also, categorizing watermelons of the whole country based on SSR polymorphism using well-chosen markers is suggested.

\section{References}

Afifia, M. D., Sayed, M. S., \& Balbaa, S. I. (1973). Nitrogenous bases of different organs of Citrullus colocynthis. Planta Medica, 24, 260-265.

Ahakpaz, F. (2000). Karyotype Analysis of local accumulations of phestocaAroundinasis. MA thesis, agriculture faculty, Isfahan Industrial University. 
Barth, A., Müller, D., \& Dürrling, K. (2002). In vitro investigation of a standardized dried extract of Citrullus colocynthis on liver toxicity in adult rats. Experimental and Toxicologic Pathology, 54(3), 223-230.

Dane, F., \& Liu, J. (2007). Diversity and origin ofcultivated and citron type watermelon (Citrullus lanatus). Genetic Resources and Crop Evolution, 54(6), 1255-1265.

Dane, F., Lang, P, \& Bakhtiyarova, R. (2004). Comparative analysis of chloroplast DNA variability in wild and cultivated Citrullus species. Theoretical and Applied Genetics, 108(5), 958-966.

Darwish, S. M., Balbaa, S. T., \& Afifi, M. S. (1974). The glycosidal content of the different organs of Citrullus colocynthis. Planta Medica, 26, 293-298.

Ghanavati, F. (2014). Assessing morphological, molecular and chemitypical various accumulations of Iranian Citrullus Colocynthis L. in terms of production anti-cancer elatrisin metabolite. MA thesis, agriculture faculty, Yasuj.

Gichimu, B. M., Owuor, B. O., Mwai, G. N., \& Dida, M. M. (2009). Morphological characterization of some wild and cultivated watermelon (Citrullus sp.) accessions in Kenya. Journal of Agricultural and Biological Science, $4(2), 10-18$.

Gichimu, B. M., Owuor, B. O., Mwai, G. N., \& Dida, M. M. (2009). Morphological characterization of some wild and cultivated watermelon (Citrullus sp.) accessions in Kenya. Journal of Agricultural and Biological Science, $4(2), 10-18$.

Goldfain, D., Lavergne, A., Galian, A., Chauveinc, L., \& Prudhomme, F. (1989). Peculiar acute toxic colitis after ingestion of colocynth: a clinicopathological study of three cases. Gut., 30, 1412-1418.

Gou, D. L., Zhang, J. P., Xue, Y. M., \& Hou, X. G. (2012). Isolation and characterization of 10 SSR markers of Momordica charantia (Cucurbitaceae). American Journal of Botany, e182-e183.

Huh, Y. C., Solmaz, I., \& Sarı, N. (2008). Morphological characterization of Korean and Turkish watermelon germplasm. In M. Pitrat (Ed.), Proceedings of the IXthEucarpiameeting on genetics and breeding of Cucurbitaceae, INRA, France, 2008

Lee, S. J., Shin, J. S., Park, K. W., \& Hong, Y. P. (1996). Detection of genetic diversity using RAPD-PCR and sugar analysis in watermelon [Citrullus lanantus (Thunb.) Mansf.] germplasm. Theoretical and applied genetics, 92(6), 719-725.

Levi, A., Claude, E. T., Keinath, A. P., \& Wehner, T. C. (2001). Genetic diversity among watermelon (Citrullus lanatus and Citrullus colocynthis) accessions. Genetic Resources and Crop Evolution, 48(6), 559-566.

Levi, A., Claude, E. T., Wehner, T. C., \& Zhang, X. (2001). Low genetic diversity indicates the need to broaden the geneticbase of cultivated watermelon. HortScience, 36(6), 1096-1101.

Moghadam, M. R. (1998). Farm and farming. Tehran University publications, 413-470.

TavakolAfshari, J., Rakhshandeh, H., Zamani, A., MahdaviShahri, N., Qazizadeh, L., Norouzi, M., \& Vahedi, F. (2005). Examining cytotoxicity of colocynth's herbal extract effect on cellular types. L929 and Hep2 magazines, 2005. Hakim, $8^{\text {th }}$ Vol., No.2, 47-54.

Ulutürk, Z. (2009). Determination of genetic diversity in watermelon (Citrullus lanatus (Thunb.) Matsum \& Nakai) germplasms.

Wang, S. Z., Pan, L., Hu, K., Chen, C. Y., \& Ding, Y. (2010). Development and characterization of polymorphic microsatellite markers in Momordica charantia(Cucurbitaseae). American Journal of Bontary, e75- e78.

Wasfi, I. A. (1994). Some pharmacological studies on Citrullus colocynthis. Journal of herbs, spices and medical plants, 2, 65-79.

\section{Copyrights}

Copyright for this article is retained by the author(s), with first publication rights granted to the journal.

This is an open-access article distributed under the terms and conditions of the Creative Commons Attribution license (http://creativecommons.org/licenses/by/4.0/). 